ISSN: $1130-3743$ - e-ISSN: 2386-5660

DOI: http://dx.doi.org/10.14201/teoredu20172912553

\title{
EL DESARROLLO HUMANO SOSTENIBLE: UN COMPROMISO EDUCATIVO
}

\author{
Sustainable buman development: \\ an educational commitment
}

\section{Le développement bumain durable: \\ un engagement éducatif}

\author{
Pilar AZNAR MíngUET* y Ángela BARRÓN RUIZ*** \\ * Universitat de València. Facultat de Filosofia i Ciències de l'Educació. \\ Departament de Teoria de l'Educació. Avda. Blasco Ibáñez, 30. 46010 València. \\ Pilar.Aznar@uv.es \\ ** Universidad de Salamanca. Facultad de Educación. \\ Departamento de Teoría e Historia de la Educación. \\ C/ Paseo de Canalejas, 169.37008 Salamanca.ansa@usal.es
}

Fecha de recepción: enero de 2017

Fecha de aceptación: marzo de 2017

RESUMEN

El desarrollo humano sostenible (en adelante DHS) se perfila como propuesta de avance ante una crisis civilizatoria de una complejidad y envergadura nada fácil de resolver. Este artículo pretende ofrecer una justificación razonada de la evolución del concepto de desarrollo y de la necesidad del compromiso educativo para el avance hacia el mismo. Si bien sigue siendo objeto de crítica y polémica, el DHS se ha consolidado como propuesta con gran fuerza ética para liderar el cambio del rumbo del desarrollo; incidiendo de forma transversal en las múltiples dimensiones del mismo y abogando por la cooperación y el diálogo interdisciplinar e intercultural. El trabajo analiza los retos que ante el DHS tiene planteada la sociedad global actual, así como 
respuestas a dar desde la acción y la investigación educativas. Concluye con una estructuración razonada del contenido del monográfico y una descripción analítica del contenido de las diferentes aportaciones.

Palabras clave: crisis civilizatoria; desarrollo humano sostenible; retos educativos; cultura de la sostenibilidad; educación para el desarrollo sostenible.

\section{SUMMARY}

Sustainable Human Development (hereafter SHD) is taking shape as a proposal for progress in the face of a crisis in civilization so complex and far-reaching that it is considered quite difficult to solve. The aim of this article is to offer a reasoned justification of the evolution of the concept of development and of the need for an educational commitment to be able to make progress towards it. Although it is still polemical and the object of criticism, SHD has become consolidated as a strongly ethical proposal to lead the change in the course of development, transversally affecting its multiple dimensions and advocating interdisciplinary and intercultural cooperation and dialogue. The article analyses the challenges posed by SHD to today's global society, as well as some ways to respond to them from the field of educational action and research. It concludes with a reasoned structuring of the contents of the monograph and an analytical description of the contents of the different contributions.

Key words: crisis of civilization; sustainable human development; educational challenges; culture of sustainability; education for sustainable development.

\section{SOMMAIRE}

Le développement humain durable (désormais DHS) s'esquisse comme une proposition d'amélioration face à une crise de civilisation d'une complexité et d'une importance difficiles de résoudre. Cet article prétend offrir une justification raisonnée de l'évolution du concept de développement ainsi que de la nécessité d'un compromis éducatif permettant l'avancement vers le DHS. Bien qu'il soit encore l'objet de critiques et de polémiques, le DHS s'est consolidé comme une proposition d'une grande force éthique pour guider le changement du cours du développement, intervenant de manière transversale dans ses multiples dimensions et plaidant en faveur de la coopération et du dialogue interdisciplinaires et interculturels. Ce travail analyse les défis posés par la société globale actuelle ainsi que les réponses possibles à partir de l'action et de la recherche éducatives. Il conclut avec une structuration raisonnée du contenu dus monographique et une description analytique du contenu des différents apports.

Mots clés: crise de civilisation; développement humain durable; défis éducatifs; culture de la durabilité; éducation pour le développement durable. 


\section{INTRODUCCIÓN}

Uno de los temas cruciales en la sociedad contemporánea es el problema de la sostenibilidad del desarrollo humano; problema asociado a un crecimiento económico que sigue la lógica de un crecimiento indefinido y no inclusivo que aumenta la vulnerabilidad, la desigualdad por razón de género, cultura o religión, la exclusión y la violencia en el interior de las sociedades y entre éstas. Los modelos de producción económica que no integran el respeto a la calidad ambiental y la cohesión y justicia social contribuyen al calentamiento global, a que se conculquen derechos humanos, así como al incremento de la intolerancia cultural, que nos lleva a un tiempo sin certezas, cambiante e imprevisible, portador de caos y ambivalencia. Las sociedades líquidas (Bauman, 2007) o del riesgo (Beck, 2008) actuales, definidas por el estado fluido y volátil que presentan, sin valores sólidos, han ido construyendo un nuevo contexto caracterizado por situaciones de dificultad social anteriormente desconocidas, en las que la incertidumbre, por la vertiginosa rapidez de los cambios, ha debilitado los vínculos humanos.

Estos problemas han adquirido un carácter global y están provocando a nivel local rápidos cambios en las estructuras sociales que ya no perduran el tiempo necesario para solidificarse, nuevas inestabilidades sociales, fuertes desequilibrios ambientales, aumento de exclusión, fragmentación social y segmentación territorial, que contribuyen a que millones de personas vivan hoy en condiciones extremas de pobreza.

En este complejo panorama, el desarrollo humano sostenible se perfila como propuesta de avance, mundialmente asumida, ante una crisis civilizatoria ${ }^{1}$ de una complejidad y envergadura nada fáciles de resolver por la humanidad.

\section{El DESARROLLO HUMANO SOSTENIBLE: UN TEMA FLUCTUANTE Y CONFLICTIVO ENTRE EL DEBATE TEÓRICO Y LA REALIDAD}

Para comprender el sentido de la primera parte del título que da nombre a este monográfico (desarrollo bumano sostenible) consideramos necesario justificar cómo, al concepto de desarrollo, se le han ido integrando los términos de humano y sostenible.

La preocupación por el desarrollo, entendida, en sentido amplio, como la preocupación por el estudio de las condiciones que favorecen el progreso y el bienestar humanos, ha acompañado a la humanidad a lo largo de su historia, y cada sociedad y cada época ha tenido su propia formulación. Sin embargo, como objeto de estudio y desarrollo teórico adquiere relevancia a partir de mediados del s. Xx,

1. Con este concepto se quiere enfatizar que estamos inmersos en una crisis de carácter integral, que afecta a dimensiones ecológicas, sociales, económicas, culturales, educativas..., y que deriva de un modelo insostenible de organización económica, productiva y de consumo que, impulsado por la globalización neoliberal, se está extendiendo en todo el planeta. 
y fundamentalmente en el campo de la Economía. Finalizada la Segunda Guerra Mundial, los países desarrollados de Europa y Norteamérica viven una época de gran prosperidad económica, en la que la demanda es superior a la producción, en un contexto de postguerra marcado por la Guerra Fría y la descolonización en Asia y África. La preocupación por mantener a los países en desarrollo en el bloque occidental alienta el desarrollo de la Economía del Desarrollo, una rama de la Economía que se dedicará a estudiar las deficiencias estructurales de los países en desarrollo y a establecer las etapas y determinantes del crecimiento económico como única vía para el desarrollo. El crecimiento económico pasará a ser considerado sinónimo de desarrollo, asumiendo que conllevará una mejora del bienestar de la población y una disminución de la pobreza (Rostow, 1961; Hirschmann, 1980).

Esta concepción del desarrollo, enmarcada en el enfoque keynesiano dominante en aquellos años, situará la clave del desarrollo en el crecimiento económico para aumentar el volumen de bienes y servicios demandados. Un crecimiento económico que vendrá de la mano del progreso tecnológico, el crecimiento de la industrialización, la urbanización y la generalización de la sociedad de consumo de masas.

El pensamiento moderno, que dio paso a la razón ilustrada y a la libertad, experimentó un gran desarrollo científico y tecnológico que fue dejando atrás creencias y prácticas que iban en contra de los supuestos racionalistas; pero, coherentemente con los planteamientos económicos, la idea lineal de progreso que se perseguía, implementada en la industrialización, basada en la producción y vinculada a la esfera económica, entroniza en exclusiva la razón instrumental, que se impone a la razón ilustrada, para aplicar la técnica a la dominación de la naturaleza por parte del ser humano, y utilizar y sobreexplotar sus recursos sin límite, llegando a definir un modelo de desarrollo basado en el crecimiento económico, considerado necesario para el bienestar humano y el aumento continuo de la calidad de vida (Habermas, 2008).

Las tesis que sustentan la concepción economicista del desarrollo se basan en un gran optimismo sobre los efectos del crecimiento, que se estimaba conllevaría grandes mejoras en la calidad de vida (salud, cultura, educación...) de los ciudadanos, y no sólo de los países más industrializados, sino que, a través de un proceso lineal, el resto de los países también accederían a dichas mejoras. Este enfoque se alzó sobre la asunción, como supuesto básico, de que el desarrollo y bienestar de las personas dependía de la riqueza global de sus países, en base a una precisa magnitud de referencia: la variación del PIB/hab. y de la renta nacional. Este planteamiento dejaba sin atender toda la problemática de la distribución de la riqueza, con lo que el desarrollo humano pasaba a ser un subproducto del desarrollo nacional (Bustelo, 1998; Sutcliffe, 1995).

Sin embargo, tal enfoque cuantitativo del desarrollo no llegó a cuantificar unos límites de crecimiento, por encima de los cuales no fueran necesarios más incrementos para alcanzar el desarrollo humano, con lo que quedaron abiertas las 
puertas para la posibilidad de un crecimiento ilimitado, sin considerar ni los límites del planeta a dicho crecimiento, ni el problema de la distribución de la riqueza. Estos efectos negativos de la modernidad industrial, economicista y cientificista, oscurecieron aquella razón emancipatoria propia de la modernidad ética impulsada por los pensadores ilustrados, y pusieron en entredicho el discurso de la ilustración al verse cuestionado en su misma racionalidad, en los usos dados a la ciencia y a la tecnología y en el propio modelo de desarrollo deseado.

Poco tiempo después, a finales de los años sesenta y principios de los setenta se empieza a tomar conciencia de que tras dos décadas de un alto crecimiento económico no se habían conseguido los resultados esperados; si bien se habían conseguido notables avances en cuanto a esperanza de vida, alfabetismo, salud, saneamiento, acceso al agua potable..., las estrategias de desarrollo puestas en marcha en aquellos años no consiguieron reducir dos grandes problemáticas del desarrollo humano, la pobreza y la desigualdad; problemáticas ambas que, por el contrario, habían aumentado.

La publicación en 1972 de Los límites del crecimiento (Meadows et al.) dejaba constancia de la existencia de un progresivo deterioro del medio natural; lo cual, unido al incremento de la pobreza y la desigualdad, apuntaba al claro fracaso de dicha concepción del desarrollo y a la necesidad de una concepción más social y menos economicista del mismo (Seers, 1969; Chenery et al., 1976; Bustelo, 1998). Así, el progreso perseguido en la modernidad basado en un modelo de crecimiento económico a ultranza no parecía propiciar un desarrollo que lograra satisfacer la calidad de vida y las necesidades humanas; y empieza a surgir, frente al desencanto por una modernidad que no ha cumplido con las expectativas deseadas, una nueva cosmovisión, como movimiento de deconstrucción de la razón instrumental. Este movimiento lleva implícito en su discurso inicial la idea de un modelo de desarrollo que cuestiona los paradigmas teóricos que habían legitimado el crecimiento económico, obviando al medio ambiente en los procesos productivos. El resultado ha sido la construcción de un nuevo paradigma centrado en el principio de sostenibilidad que trata de eliminar la contradicción aparente entre crecimiento económico, conservación de la naturaleza y satisfacción de las necesidades humanas presentes y futuras; lo cual, a día de hoy en la práctica, se ha quedado en un mero discurso.

Este pensamiento, llamado postmoderno, subraya los límites de la ciencia a la generación de conocimiento verdadero, acumulativo y de validez universal, renuncia a las utopías y a la idea de progreso de conjunto, apostando a la carrera por el progreso individual, al mismo tiempo que se va produciendo en el orden económico capitalista el cambio de una economía de producción a una economía del consumo (Lyotard, 1989; Vattimo Vigo, 1986). En este proceso de cambio, el pensamiento postmoderno tropieza con nuevos problemas: los medios de comunicación de masas, la globalización de los mercados y la industria del consumo masivo se convierten en centros de poder, primando el crecimiento económico como estrategia política ante la crisis social, económica y ambiental. 
Bajo el impulso de Naciones Unidas se promueve, a partir de los años setenta, una revisión de tales planteamientos ortodoxos, reivindicándose otras propuestas en la concepción del desarrollo, más preocupadas por el desarrollo social y por una redistribución más equitativa de la riqueza. Desde entonces hasta la actualidad han sido reiterados y conflictivos los debates en torno a dicha temática; hasta el punto de que para algunos autores postdesarrollistas y postmodernos no tiene sentido seguir insistiendo en torno a la idea del desarrollo como noción universal, sino que más bien será necesario profundizar en las distintas visiones locales del bienestar humano, y así evitar la expansión de un modelo hegemónico que responde a los intereses de la civilización occidental (Latouche, 2007). Sobre estos presupuestos se han desarrollado enfoques alternativos que proponen alternativas para un desarrollo a escala humana con énfasis en lo local, como los modelos de desarrollo endógeno basados en el empoderamiento de la ciudadanía para potenciar las capacidades internas de las comunidades locales con el objetivo de impulsar un desarrollo comunitario sostenible.

Sin embargo, más allá de este planteamiento, los principales debates sobre el desarrollo suelen enmarcarse en un terreno fluctuante y conflictivo entre las concepciones teóricas y las realidades prácticas por adaptarse a las nuevas demandas conceptuales, pero sin alterar la estrategia economicista, que, apoyada desde la década de los años noventa por la globalización neoliberal, se resiste a cambiar de rumbo y muy por el contrario parece decidida a seguir en una huida hacia adelante, hasta el punto de que hoy en día la idea de progreso basada en el crecimiento económico parece seguir vigente en la práctica y el discurso político dominante.

Así parece que el pensamiento postmoderno tampoco está consiguiendo cumplir con las expectativas deseadas; lo que ya ha motivado el despliegue de discursos en busca de vías alternativas que recuperen, reeditando y revisando los conceptos de progreso vigentes. En esta búsqueda se apela al imperativo de la razón humana frente a la razón instrumental y a la recuperación crítica de los valores de la modernidad y se defiende que, para recuperar los valores de la modernidad, es necesario construir un nuevo humanismo y afrontar una reconceptualización de la racionalidad que propugne un desarrollo basado en la aplicación de la ciencia y la tecnología al servicio de la humanidad y con respeto a los valores de la sostenibilidad (Habermas, 2008; Riechmann, 2006).

Es así como en el discurso teórico se observa la existencia de diversos intentos por revisar la concepción del desarrollo, asumiendo los límites del planeta, así como la existencia de problemas globales e interdependientes que ponen en grave riesgo el bienestar humano y la comunidad biótica (calentamiento global, pérdida de biodiversidad, degradación de ecosistemas, desertificación de suelos, incremento de las desigualdades...). En la búsqueda de estos planteamientos han tenido especial incidencia algunos elementos clave como son: una nueva consideración del bienestar y el progreso humano, la necesidad de considerar también el bienestar de las futuras generaciones y la existencia, en el planeta, de una base limitada de recursos recursos (Novo, 2006). En este sentido, para la conformación de 
una nueva concepción del desarrollo fue clave la aportación del premio Nobel de Economía de 1998, Amartya Kumar Sen, con su crítica al utilitarismo convencional y la propuesta de un nuevo enfoque centrado en las personas. Enfoque que fue adoptado, desde 1990, por Naciones Unidas para elaborar sus programas e informes sobre Desarrollo Humano. Los trabajos de Sen sobre la desigualdad, el desarrollo y la libertad, en la década de los 70, ayudaron a conformar el concepto de ética del desarrollo y proponer temas como la libertad y la justicia en los estudios técnicos del desarrollo. Gracias a sus aportaciones tomó forma el enfoque de las "capacidades" como indicador de desarrollo humano; un enfoque en el que se apoyará la nueva concepción del desarrollo centrado en las personas, que será impulsada por Naciones Unidas con la aparición en 1990 del primer Informe Mundial sobre Desarrollo Humano ${ }^{2}$ (Sen, 1995; PNUD, 1990; Nussbaum, 2002; Cortina y Pereira, 2009).

Es así como se conforma el concepto de «Desarrollo Humano». El capítulo 1 de dicho primer Informe Mundial, dedicado a la «Definición y medición del desarrollo humano", se inicia con estas palabras:

La verdadera riqueza de una nación está en su gente. El objetivo básico del desarrollo es crear un ambiente propicio para que los seres humanos disfruten de una vida prolongada, saludable y creativa. Esta puede parecer una verdad obvia, aunque con frecuencia se olvida debido a la preocupación inmediata de acumular bienes de consumo y riqueza financiera (PNUD, 1990, 31) .

A partir de este primer Informe, el discurso sobre el desarrollo humano considerará el crecimiento económico como un medio, no un fin en sí mismo, y el punto de mira se centrará en aumentar la riqueza de la vida humana, en dar a las personas más libertad, oportunidades y capacidades para vivir una vida que valoren. En la obra de Sen, Desarrollo y libertad (2000), la libertad pasará a ser considerada como el principal objetivo del desarrollo humano, a la vez que su medio principal. El conjunto coordinado de sistemas democráticos, jurídicos, educativos, sanitarios, de mercado, comunicación, etc., deberán apoyar los medios (económicos, libertades políticas, oportunidades sociales, seguridad,

2. En 1989, y a iniciativa de la Organización de las Naciones Unidas, se constituyó un equipo de trabajo, conformado por personal del PNUD y eminentes consultores externos, bajo la coordinación de Mahbub ul Haq, exministro de Hacienda y Planeación de Pakistán, con la intención de preparar un informe sobre el emergente concepto de desarrollo humano. Entre el grupo de consultores estaba Amartya K. Sen.

3. Desde que en 1990 apareció el primer informe sobre desarrollo humano, se han ido emitiendo informes anuales todos los años, en forma de análisis independientes acerca de cuestiones, tendencias y políticas del desarrollo (http://hdr.undp.org/). En todos ellos se sigue manteniendo la misma concepción del desarrollo humano, y así se afirma en el inicio del Prólogo del último informe sobre Desarrollo Humano (2016): «El desarrollo humano tiene por objeto las libertades humanas: la libertad de desarrollar todo el potencial de cada vida humana -no solo el de unas pocas ni tampoco el de la mayoría, sino el de todas las vidas de cada rincón del planeta- ahora y en el futuro» (PNUD, 2016, III). 
transparencia...) para el ejercicio de la libertad, de modo que se haga efectivo el objetivo del desarrollo humano, entendido como ampliación de las oportunidades del ser humano y mejora de sus capacidades y libertades. Oportunidades que pueden ser infinitas y cambiar con el tiempo, pero entre las que pueden subrayarse tres esenciales: la oportunidad de disfrutar de una vida prolongada y saludable; la de tener acceso a la educación y adquirir conocimientos, y la oportunidad de tener acceso a los recursos para disfrutar de un nivel de vida decente. De acuerdo con ello, a la hora de medir el desarrollo humano, en dicho primer Informe se pondrá de manifiesto la inadecuación de los indicadores convencionales (PIB, renta nacional), y en su lugar se establecerán tres indicadores claves: la esperanza de vida al nacer, la tasa de alfabetización y la renta per cápita, aun reconociendo la necesidad de tener en cuenta otros aspectos difíciles de medir pero necesarios de considerar, como la libertad política, la participación en la comunidad, la seguridad y los derechos humanos, la igualdad entre hombres y mujeres, la sostenibilidad medioambiental (PNUD, 1990, 36-39). Con este cambio de enfoque han ido surgiendo otros indicadores, como el índice de felicidad interior bruta de una comunidad, que abren la puerta a que, con mayor o menor habilidad, se planteen otras opciones a la hora de valorar la riqueza de un país y el bienestar de sus ciudadanos (Helliwell et al., 2017).

A este avance hacia una concepción del desarrollo centrado en las personas en lugar de en el crecimiento económico, se unirá la perspectiva de la sostenibilidad, aportada por el conocido como Informe Brundtland (WCED, 1987); en cuyo apartado sobre El desarrollo duradero plantea lo siguiente: «Está en manos de la humanidad hacer que el desarrollo sea sostenible, duradero, o sea, asegurar que satisfaga las necesidades del presente sin comprometer la capacidad de las futuras generaciones para satisfacer las propias» (WCDE, 1987, 23), y para ello, en la página 13, propone: «Lo que se necesita ahora es una nueva era de crecimiento económico, un crecimiento que sea poderoso a la par que sostenible social y medioambientalmente» ${ }^{4}$.

Aparece así el término sostenible, que se unirá al de desarrollo humano para conformar la expresión Desarrollo Humano Sostenible que da título a este monográfico, y que será asimismo adoptada por Naciones Unidas, en el Informe Mundial sobre Desarrollo Humano que publicó en 1992. En el cap. 1 de dicho Informe aparece ya, por primera vez, un apartado con el título "Desarrollo humano sostenible», aunque no da una definición del mismo, sino que asume la definición de desarrollo

4. La toma de conciencia de la acelerada degradación medioambiental, así como del incremento de las desigualdades sociales y las consecuencias de todo ello en el desarrollo humano, fue la causa de que la Asamblea General de Naciones Unidas creara en 1983 la Comisión Mundial del Medio Ambiente y del Desarrollo, presidida por la primera ministra noruega Gro Harlem Brundtland. Tras numerosos encuentros participativos por todo el planeta, esta Comisión presentó a la Asamblea General, en 1987, el Informe "Nuestro Futuro Común», más conocido como Informe Brundtland (WCED, 1987), en el que reconociendo la insostenibilidad del viejo enfoque economicista del desarrollo se urge a avanzar hacia un desarrollo sostenible. 
sostenible del Informe Brundtland (1987), insistiendo en el principio básico de colocar a las personas en el centro del desarrollo (PNUD, 1992, 40-50). Habrá que esperar al Informe Mundial de 1994 para encontrar en el Prefacio del mismo la siguiente definición: "El desarrollo humano sostenible es el desarrollo que no sólo suscita un crecimiento económico, sino que también distribuye equitativamente sus beneficios; que regenera el medio ambiente en lugar de destruirlo; que fomenta la autonomía de las personas en lugar de marginarlas» (PNUD, 2014, iii). Más adelante, se plantea la necesidad de «un nuevo paradigma del desarrollo que coloque al ser humano en el centro del desarrollo, considere el crecimiento económico como un medio y no como un fin, proteja las oportunidades de vida de las futuras generaciones al igual que las de las generaciones actuales y respete los sistemas naturales de los que dependen todos los seres vivos» (PNUD, 1994, 4-5).

La propuesta de cambio hacia un desarrollo humano sostenible (en adelante DHS) ha pasado a constituir el punto de inicio de una intensa discusión, sin precedentes en el debate internacional y todavía vigente, acerca de la delimitación conceptual del mismo, sobre el modo de abordar el reto de la sostenibilidad en el desarrollo humano, así como acerca de la propia concepción del término "necesidades»; ya que el Informe Brundtland induce a significados políticamente confusos, inciertos, cambiantes, imprevisibles y ambivalentes, puesto que habría que precisar cuáles son las necesidades esenciales a satisfacer, más allá de los planteamientos teóricos clásicos que defienden que las necesidades son infinitas y cambiantes en el tiempo y en el espacio. La nueva teoría de las necesidades fundamentales (Max Neef, 1993; Doyal y Gough, 1994) defiende la tesis de que las necesidades humanas son finitas y clasificables, idénticas en las diferentes culturas y no cambian con el tiempo; lo que realmente cambia es la manera o los medios utilizados para la satisfacción de las mismas. Desde esta óptica, el desarrollo sostenible estará en función no tanto de la modificación de las necesidades humanas fundamentales, sino del cambio que se pueda producir en el uso de satisfactores o formas mediante las cuales las necesidades sean satisfechas, teniendo en cuenta el modelo sinérgico según el cual la satisfacción de una de ellas contribuye a la satisfacción simultánea de otras, y sin perder de vista los límites que conlleva su satisfacción; que vienen marcados por la cantidad y variedad de recursos disponibles en el sistema que mantiene la vida en el planeta (Elizalde, 2009, 71; Aznar y Ull, 2012, 71).

Entre los argumentos críticos más comúnmente utilizados frente al DHs figuran: la vaguedad en su delimitación conceptual, que da lugar a muy variadas interpretaciones al servicio de intereses muchas veces contrapuestos (Hopwood, Mellor y O’Brien, 2005); las contradicciones semánticas que surgen de la unión entre crecimiento económico poderoso y preservación ecológica, dada la difícil conciliación entre ambos (Lafferty y Meadowcroft, 2000; García, 2004); la dificultad de hacer operativo el cambio de modelo, frente a la resistencia que ejerce la globalización neoliberal dominante, dada la amplitud, complejidad y alcance que supone el cambio de rumbo hacia la sostenibilidad (Barbiroli, 2009; López, 2008); la perspectiva 
antropocéntrica que presenta, al centrar el punto de mira en la satisfacción de las necesidades humanas y no en una perspectiva más biocéntrica, lo que ha dado lugar a diversas acepciones más «fuertes» o más «débiles» de la sostenibilidad (Gudynas, 2004; Naredo, 2006), etc.

Sin embargo, el reiterado problema de vaguedad y ambigüedad conceptual que afecta a la definición del «desarrollo sostenible» es interpretado por varios autores como el principal atractivo del término ya que puede servir para cualquier cosa, y entre otras para haber generado un dilatado consenso internacional en torno a la necesidad del cambio de rumbo del desarrollo en pro de la sostenibilidad, que debe asumir la agenda política nacional e internacional, en el marco del proceso de homogeneización de la sociedad global en la que estamos inmersos (Dale, 2001; Jordan y Adger, 2009). Ello explica que desde que se firmara la Declaración de Río y la Agenda 21 en la Cumbre de Río (1992), pasando por las cumbres posteriores (Johannesburgo, 2002; Río, 2012), la propuesta del Desarrollo Sostenible haya obtenido el apoyo necesario para extender en todo el mundo la necesidad de una nueva concepción del desarrollo que sirva como base para el consenso mundial, y que ha llevado a Naciones Unidas al establecimiento en septiembre de 2015 de la Agenda 2030 para el desarrollo sostenible (ONU, 2015).

Una propuesta, la del cambio de rumbo hacia la sostenibilidad, que, si bien sigue siendo objeto de crítica y polémica, se ha consolidado como noción abstracta con gran fuerza ética para liderar el cambio de rumbo del desarrollo, adquiriendo su legitimidad al conformar una nueva visión global de futuro, democráticamente consolidada y universalmente aceptada, que incide de forma transversal en todos los ámbitos de la vida social (político, económico, social, cultural, científico-tecnológico, educativo...) y aboga por la cooperación y el diálogo interdisciplinar e intercultural.

\section{NATURALEZA MULTIDIMENSIONAL DEL DESARROLLO HUMANO SOSTENIBLE (DHS) Y LA IMPORTANCIA DEL COMPROMISO EDUCATIVO}

El DHS se caracteriza por constituir una propuesta sistémica y glocal de desarrollo, basada en principios éticos como el respeto al funcionamiento de los ciclos naturales y la diversidad biológica y cultural, así como la promoción de la equidad social y los derechos humanos, en el marco de democracias participativas. Una propuesta en la que los objetivos económicos están subordinados al respeto de las leyes del equilibrio ecológico y a la mejora de la calidad de vida de todas las generaciones presentes y futuras. Requiere, por tanto, contemplar no sólo la dimensión del crecimiento económico, medio sin duda indispensable, sino también otras dimensiones que de forma interdependiente la conforman, como son la dimensión ecológica, política, social, demográfica, cultural, científico-tecnológica, educativa... (Colom, 2007; Duarte, 2006; Murga, 2015; Rieckmann, 2005).

En lo económico, dimensión habitualmente enfrentada a lo ecológico, el DHS reclama la reconceptualización de la economía y del mercado como medios para el 
desarrollo y no como fines del mismo ${ }^{5}$. Este planteamiento obliga a hacer frente a la ideología neoliberal dominante, de modo que los frutos del crecimiento se inviertan en promover el bien común y ampliar las oportunidades para que las personas vivan mejor, y no en seguir maximizando las brechas y desigualdades sociales y el deterioro del medio natural. Hacer frente a la extensión del capitalismo neoliberal no es empresa fácil, dado que es un sistema que tiende a la búsqueda de la plusvalía y el crecimiento continuado, guiado por los intereses de las grandes corporaciones empresariales, comerciales y financieras; pero si bien no es fácil, cada vez es una prioridad más necesaria si queremos evitar el colapso al que conducen las desajustadas relaciones humanas y de los humanos con la biosfera. Ello obliga a regular de otro modo los mercados y reformular los modos de extracción, producción, distribución y consumo, así como a transformar las concepciones comerciales y financieras dominantes por otras más sostenibles, como son el comercio justo, la economía solidaria, las finanzas éticas, la economía circular, la economía del bien común ${ }^{6}$, etc.; propuestas todas ellas ya consolidadas y bien fundamentadas en la ética de la sostenibilidad, aunque poco apoyadas y generalizadas (Diamond, 2006; Fernández, 2004; Felber, 2012; Jackson, 2011; Sachs, 2008; Stiglitz y Charlton, 2007).

En lo ecológico, el DHS plantea la necesidad de respetar la preservación de los recursos naturales, el equilibrio de los ecosistemas y el mantenimiento de la biodiversidad; lo que permitirá la perpetuación en el tiempo de los recursos necesarios para proporcionar calidad de vida a una población en expansivo crecimiento demográfico. Condiciones que obligan a transformar el modelo económico, así como el sistema productivo y de consumo hacia perspectivas de sostenibilidad, reconociendo los límites al crecimiento que impone el sistema ecológico, cuyos recursos no son ilimitados, así como también es limitada la capacidad de carga de los ecosistemas para restablecerse de los impactos antrópicos generados. El reconocimiento de tales límites obliga a descartar la posibilidad de un crecimiento

5. Los informes de Naciones Unidas para el Desarrollo no dejan de reiterar año tras año esta idea. Así lo expresa, por ejemplo, el informe de 1993, cuando dice: «El desarrollo humano sostenible exige más de los mercados: que sigan brindando sus ventajas pero que lo hagan de forma más equilibrada, combinando la eficiencia, la equidad y la sostenibilidad. Después de todo, los mercados no son un fin en sí mismos. Son un medio para el desarrollo humano. Los mercados deben estar al servicio de la gente. La gente no debe estar al servicio de los mercados» (PNUD, 1993, 36).

6. Desde que en 1989 apareciera la obra de Herman Daly y John Cobb, con el título For the common god. Redirecting the economy toward community, the environment, and a sustainable future, esta necesidad de reconceptualizar la Economía y el Mercado al servicio del bien común no ha dejado de ser reivindicada. La publicación en 2010 de la obra de Christian Felber Die Gemeinwohl-Ökonomie, publicada en España en 2012 con el título La economía del bien común (FELBER, 2012), ofrece una propuesta de transformación del capitalismo neoliberal a la economía del bien común, en la que el dinero, el capital y el beneficio financiero se transforman en medios al servicio de dicha finalidad; a la vez que se ofrece el modo de hacer operativa dicha propuesta a través del "balance del bien común", que mide el éxito de las empresas, y el "producto del bien común", para medir el éxito de las economías nacionales. Una propuesta a la que ya se han adherido bastantes empresas, universidades, municipios..., y que cuenta con organizaciones sociales en muchos países (http://www.economia-del-bien-comun.org/es). 
económico continuado. Si bien son muchos los avances realizados en dicha dirección, tales como el reciente acuerdo de París para reducir las emisiones de gases de efecto invernadero ${ }^{7}$, los múltiples programas de gestión de residuos y del medio natural, las diversas normas y reglamentos para prevenir y corregir los impactos ambientales de los proyectos y actividades empresariales, comerciales y productivas...; sin embargo, aún son muchas las voces críticas que cuestionan la dificultad de armonizar un sistema económico globalizado y neoliberal, en continuo crecimiento, con los límites que exige el respeto a los ciclos de la naturaleza (Jackson, 2011; Meadows, Randers y Meadows, 2006; Sachs, 2008).

En lo social, el DHS plantea la prioridad ética de erradicar la pobreza, reducir las desigualdades sociales, promover la justicia y la equidad, fortalecer la identidad de los pueblos, los derechos humanos y cuidar el equilibrio demográfico. Todo lo cual demanda una mejor distribución del crecimiento económico de modo que las generaciones presentes y futuras puedan tener oportunidades para mejorar su calidad de vida. Ello plantea la necesidad de establecer límites inferiores y superiores, tanto en lo relativo a la explotación de recursos que realizan los sistemas productivos, con el fin de mantener los equilibrios ecológicos, como en lo que hace referencia al consumo de recursos y energía que realizan los grupos sociales, que deberán mantenerse por encima del límite de pobreza y por debajo del límite de la opulencia, con el fin de asegurar los equilibrios sociales y la satisfacción de las necesidades humanas. Asimismo, la prioridad de erradicar la pobreza supone que el poder político asuma como criterios básicos de su política pública la justicia distributiva y la universalización de la cobertura de los derechos humanos, como son los de salud, alimentación, educación, información, representación, vivienda, seguridad, etc.

En lo político, el DHS aspira a fortalecer y profundizar la democracia y la construcción de la ciudadanía, promoviendo y garantizando la participación ciudadana en la gestión del desarrollo y en la toma de decisiones públicas, así como reivindicando el respeto de los derechos humanos y el cumplimiento de los deberes ciudadanos. A este respecto, los poderes políticos deben acordar un mayor poder de regulación de los intereses de las corporaciones económicas, comerciales y financieras para situar en el centro de interés el DHS, y con ello la defensa del equilibrio ecológico, la equidad social y la calidad de vida de la población. Ello requiere de Estados fuertes con capacidad para planificar, informar y gestionar con transparencia y responsabilidad ante el control ciudadano, poniendo el crecimiento económico al servicio del bien común; y asimismo también requiere fortalecer la

7. El histórico Acuerdo de París sobre el cambio climático da testimonio del consenso logrado por la humanidad ante dicho desafío mundial. Un consenso impensable en épocas anteriores y que, sin embargo, ha sido adoptado el 12 de diciembre de 2015 en el marco de la XxI Conferencia sobre Cambio Climático (COP 21), y ha entrado en vigor el 4 de noviembre de 2016, al ser ratificado por 55 países que suman el 55\% de las emisiones globales. 
capacidad de las organizaciones sociales y ciudadanas para acceder a la información, controlar la gestión del gobierno y participar en la toma de decisiones.

En lo cultural, el DHS reivindica un cambio en los patrones culturales de relación entre los humanos y de éstos con la naturaleza de modo que lleguemos a conseguir un manejo sostenible de los ecosistemas y los recursos naturales, a la vez que un respeto a la diversidad cultural; un impulso a la participación ciudadana en la gestión del desarrollo, y una distribución equitativa de los recursos y servicios para resolver las injusticias sociales y satisfacer las necesidades y derechos humanos. Los patrones culturales deben evolucionar hacia estilos de producción y consumo sostenibles, así como estilos de relación interpersonal respetuosos con la diversidad cultural y solidarios con las generaciones presentes y futuras.

En la sociedad global contemporánea, el cuestionamiento de la racionalidad neoliberal y el avance en todas las dimensiones que sustentan la propuesta, sistémica y global, del DHs debe ser asumido y acordado, de manera colaborativa y transversal por parte de todos los actores implicados, desde los organismos internacionales y gobiernos, las grandes corporaciones y pequeñas empresas locales, las universidades y centros de enseñanza..., hasta llegar a los diversos sectores productivos, entidades sociales y el compromiso de todos los ciudadanos (Stiglitz, 2006).

Para conseguir el compromiso de todos los sectores implicados en la propuesta del DHs, es fundamental que la educación y formación para la sostenibilidad llegue a todos ellos y consiga transformar la cultura dominante. Para ello, el compromiso educativo del Sistema Educativo, Formal y No Formal, es de importancia capital. Así se planteó ya en 1975 cuando se redactó el marco y directrices de la Educación Ambiental (en adelante EA) en la Carta de Belgrado estableciendo que:

La reforma de los procesos y sistemas educacionales es el núcleo para la edificación de esta nueva ética del desarrollo y del nuevo orden económico mundial. Los gobiernos y los encargados de decidir las políticas pueden ordenar que se realicen cambios, al tiempo que nuevos sistemas de desarrollo pueden comenzar a mejorar la condición mundial, pero todo ello sólo representa una solución por breve plazo, a menos que la juventud del mundo reciba un nuevo género de educación ${ }^{8}$.

Desde entonces hasta la actualidad, la EA ha ido integrándose con más o menos éxito en los sistemas educativos de todo el panorama internacional, de modo más acertado en el ámbito No Formal que en el Formal, en el que todavía

8. http://unesdoc.unesco.org/images/0001/000177/017772sb.pdf, 2 La comúnmente conocida como Carta de Belgrado, que lleva el título de "Un marco general para la educación ambiental», constituye la Declaración del Seminario Internacional de Educación Ambiental, celebrado del 13 al 22 de octubre de 1975, en el marco del programa PNUMA de la UNESCO. En dicha Carta se definen las metas, objetivos, destinatarios y principios de la Educación Ambiental; a la que se considera de importancia capital para lograr la formación de una nueva ética universal que consiga reorientar el enfoque dominante del desarrollo centrado en aumentar al máximo el rendimiento económico, por otro enfoque que busque mejorar la calidad del medio y la calidad de vida para todos cuantos viven y vivirán en ese medio. 
sigue siendo un objetivo a conseguir su integración transversal. Desde la década de los 70 hasta la actualidad, en los diversos programas y actividades de EA se han trabajado de forma predominante los aspectos naturales del medio ambiente, con planteamientos próximos a la ecología y a las ciencias naturales y desde enfoques más disciplinares que inter- o transdisciplinares. Desde la década de los 90 y tras la celebración de la Cumbre de Río se insiste en la necesidad de reforzar la orientación de la EA hacia la promoción del desarrollo sostenible, incorporando al estudio de los elementos naturales las dimensiones socioculturales (políticas, económicas, sociales, educativas...) para poder entender el modo tan desajustado en que la humanidad ha gestionado las relaciones entre los pueblos y de éstos con la naturaleza, y reorientar dichas relaciones hacia la ética ecológica (Barrón, 2002; Caride y Meira, 2001). Estos nuevos enfoques de EA para el desarrollo sostenible integran la preocupación tradicional por el uso sostenible de los recursos (agua, energía, residuos, flora, fauna...); por la reducción de la pobreza y las desigualdades sociales; por la universalización de los derechos humanos; por el comercio y las finanzas éticas; por un consumo responsable; por un desarrollo urbanístico, científico y tecnológico sostenibles; por el avance hacia democracias más participativas y una mejor gobernanza mundial; por la responsabilidad social de las empresas, el respeto a la diversidad cultural y la paz; por una economía solidaria del bien común, etc. (Aznar y Ull, 2012; Murga, 2015; Tilbury, 2011; Vilches, Macías y Gil, 2014).

Impulsados por la UNESCO, el Decenio de la Educación para el Desarrollo Sostenible (2005-2014) y como continuación del mismo, el Programa de Acción Global (GAP) de EDS (2015-2030) plantean con claridad la importancia del compromiso educativo con la sostenibilidad en todos los niveles y contextos sociales, y así figura en la web de la UNESCO, cuando expresa: "El desarrollo sostenible no se logra únicamente con soluciones tecnológicas, medidas políticas y recursos económicos: también debemos cambiar nuestra forma de pensar y actuar. Para conseguirlo, se necesita una educación para el desarrollo sostenible de calidad a todos los niveles y en todos los contextos sociales»?

La educación para el desarrollo sostenible (en adelante EDS) se convierte así en algo tan importante como leer o escribir, un eje transversal que deberá atravesar la forma de pensar y actuar de la humanidad en todos los ámbitos ocupacionales, con la finalidad de regular nuestras relaciones con el medio natural y social en base a la ética de la sostenibilidad como vía para avanzar, entre todos, hacia un DHS (UNESCO, 2014a, 2014b, 2015; UNECE, 2011, 2013).

La ética de la sostenibilidad que sustenta esta propuesta de desarrollo ha quedado recogida en la Carta de la Tierra, tras el proceso de diálogo intercultural más inclusivo y participativo jamás logrado en la creación de un marco ético. La redacción de esta Carta, a iniciativa de Naciones Unidas, concluyó en el año 2000, tras una década de debates a nivel mundial, y ofrece un marco ético para orientar

9. Disponible en https://en.unesco.org/themes/education-sustainable-development. 
la transición hacia el DHs, en el que se establecen cuatro principios éticos: 1) el respeto y cuidado de la comunidad de vida; 2) la integridad ecológica; 3) la justicia social y económica; y 4) la democracia, no violencia y la paz; reconociendo que «los objetivos de la protección ecológica, la erradicación de la pobreza, el desarrollo económico equitativo, el respeto a los derechos humanos, la democracia y la paz son interdependientes e indivisibles» ${ }^{10}$. Estos principios están diseñados para servir de base desde la que guiar y evaluar la conducta de las personas, organizaciones, empresas, gobiernos e instituciones transnacionales (Murga-Menoyo, 2009).

Si bien existe un alto consenso internacional acerca de la naturaleza holística y multidimensional de esta propuesta de desarrollo y la importancia capital del compromiso educativo para su consecución, la realidad es que su avance en la práctica es todavía incipiente, dado que la transición a un DHS requiere de un avance coordinado en los diversos factores glocales (globales y locales a la vez) que estrechamente interconectados conforman tal propuesta integral de desarrollo. Como diversos autores señalan es necesaria un auténtica "(r)evolución por la Sostenibilidad, que muestra acertadamente la necesidad de unir los conceptos de revolución y evolución: revolución para señalar el cambio profundo, radical, en nuestras formas de vida y organización social; evolución para puntualizar que no se puede esperar tal cambio, fruto de una acción concreta, más o menos acotada en el tiempo» (Vilches, Macías y Gil Pérez, 2014, 12).

\section{RetOs EDUCATIVOS ANTE EL PROBLEMA DE LA SOSTENIBILIDAD DEL DESARROLLO HUMANO}

Esta r-evolución por la sostenibilidad, en una sociedad global que transforma el contexto social y cultural en que tradicionalmente se ha desarrollado la educación, viene planteando importantes retos educativos, ante los que se están generando avances en la acción y en la investigación, que ponen en cuestión enfoques pedagógicos tradicionales.

Tres aspectos resultan especialmente significativos: i) Redefinición de nuestra relación con el medio natural/humano/social, tanto a nivel de representación como de recreación, con miras a un desarrollo humano sostenible. ii) Dualidad entre lo estándar/global/colectivo y lo único/personal/individual, estableciendo una necesaria interacción entre los referentes globales y las actuaciones locales. iii) Redefinición de los modelos curriculares desde la óptica de la sostenibilidad.

10. Disponible en: http://cartadelatierra.org/descubra/que-es-la-carta-de-la-tierra/. La Carta de la Tierra, que surgió como iniciativa de Naciones Unidas, concluyó sin embargo como iniciativa de la sociedad civil. La Comisión de la Carta de la Tierra, una entidad internacional independiente, la hizo pública como una carta de los pueblos el 29 de junio en el Palacio de Paz, de La Haya, en Holanda. Dado el proceso participativo de elaboración y el respaldo obtenido por más de 6.000 organizaciones internacionales se considera, jurídicamente hablando, como una ley blanda, base para el futuro desarrollo de una ley vinculante. 
Estos aspectos, que son objeto de tratamiento científico por los autores de los artículos en este monográfico, están presentes de forma reiterada en los documentos publicados en los últimos cinco años por los organismos internacionales en materia de educación.

En primer lugar, la Estrategia unECE de Educación para el Desarrollo Sostenible de Naciones Unidas, "Empowering educators for a sustainable future» (UNECE 2013), propone una redefinición de la formación de formadores con el objetivo de proporcionar a los profesionales en educación conocimientos, habilidades, actitudes y valores compatibles con los valores de la sostenibilidad; y de acuerdo a este objetivo ha establecido un marco de competencias para el desarrollo profesional de los educadores que realizan sus funciones tanto en los ámbitos formales, como en los no formales e informales. El marco de competencias propuesto se basa en los cuatro pilares del Informe Delors (1997): aprender a conocer, aprender a hacer, aprender a convivir y aprender a ser, adaptados al ámbito de la sostenibilidad. Tal marco de competencias requiere un enfoque holístico que integre el pensamiento y la acción, una concepción dinámica de la realidad y un objetivo de transformación de la sociedad hacia un desarrollo sostenible. Desde esta óptica el documento ofrece ejemplos de talleres con un conjunto de actividades que puede servir como base para la realización de prácticas de formación adaptables a diversas circunstancias particulares y contextos; así como modelos de evaluación basados en los resultados de los aprendizajes esperados.

También el Programa de Acción Global (GAP), impulsado por la UNESCO (2014b) en la "Declaración sobre la Educación para el Desarrollo Sostenible», promueve la educación en todos los niveles como un importante activo para implementar la sostenibilidad en todas sus áreas de acción, y considera la transición a la sostenibilidad como uno de los más importantes retos actuales tanto a niveles globales como locales. La transición a la sostenibilidad no puede verse como una apuesta de futuro, sino como una estrategia a aplicar en el presente para construir un futuro viable, desde la integración de tres cadenas de pensamiento: la que busca la calidad ambiental, la que busca la cohesión y la justicia social y la que busca una economía equitativa y viable a largo plazo (CRUE 2012). Éste es el compromiso renovado por la educación para la sostenibilidad, contenido en este documento, desde el cual se urge a la toma de medidas urgentes para fortalecer y ampliar en mayor medida las acciones educativas dirigidas a la consecución de un desarrollo humano inclusivo, ambiental y socialmente sostenible.

Por otra parte, como complemento a los informes mundiales sobre la educación (Faure, 1972; Delors, 1996), la directora general de la UnESCO, Irina Bokova, formó un grupo de expertos para que se replanteara la educación en un mundo en proceso de cambio. El resultado ha sido la publicación de un documento (UNESCO, 2015) cuyo primer capítulo, "El desarrollo sostenible: una preocupación esencial», representa una invitación a la búsqueda de respuestas ante los retos de afrontar la sostenibilidad económica, social y ambiental. El texto reinterpreta los planteamientos de los anteriores informes mundiales sobre la educación, y los replantea desde 
la óptica del bien común mundial. La consideración de la educación como un «bien común" transciende la idea contenida en la teoría socioeconómica individualista inherente a la noción de "bien público", al centrarse en un proceso participativo a la hora de definir lo que es un bien común que tome en consideración la diversidad de contextos, de conceptos de bienestar y de cosmovisiones. Este documento está inspirado en una concepción humanista de la educación y el desarrollo, y en la defensa de una educación inclusiva, "basada en el respeto a la vida y la dignidad humana, la igualdad de derechos, la justicia social, la diversidad cultural, la solidaridad internacional y la responsabilidad compartida de un futuro sostenible» (UNESCO, 2015 , 9). En este modelo de educación, el crecimiento económico precisa balancearse con la búsqueda de la calidad ambiental y la justicia social y exige reconceptualizar la educación y el conocimiento como bienes comunes mundiales, así como su función en el desarrollo humano, basado en fundamentos éticos y morales renovados; ya que el modelo de desarrollo que impulsa la economía global torna necesario reconducir las formas de vida que inciden en el consumo insostenible de recursos, la degradación de los ecosistemas y la explotación de las personas, por otro modelo que preserve el bienestar humano sin exceder los límites del planeta.

En el mismo año, en la Cumbre de Naciones Unidas (25-27 septiembre de 2015) celebrada en Nueva York se aprueba el documento "Transformar nuestro mundo: la Agenda 2030 para el Desarrollo Sostenible», que presenta respuesta globales a los retos que tienen planteados las sociedades actuales partiendo de la base de que la erradicación de la pobreza en todas sus formas y dimensiones, la lucha contra la desigualdad dentro de los países y, entre ellos, la creación de un crecimiento económico inclusivo que preserve la calidad ambiental y fomente la cohesión social están vinculados entre sí y son interdependientes (ONU, 2015). El documento actualiza y amplía los Objetivos de desarrollo del milenio y relanza una Agenda universal para la transición hacia un desarrollo durable con vistas al 2030, estableciendo 17 Objetivos de Desarrollo Sostenible y 169 metas a desarrollar fundamentalmente en tres esferas: las personas, el planeta, la prosperidad, y a través de dos medios: la paz y las alianzas. De forma específica, el objetivo n. ${ }^{\circ} 4$ se refiere a que todos los alumnos adquieran los conocimientos y competencias necesarias para promover un desarrollo viable ecológicamente y equitativo humana y socialmente. Y recomienda que las instituciones educativas en general y la universidad en particular incorporen a la docencia, además de los conocimientos científicos y técnicos, las competencias y capacidades genéricas y transversales vinculadas a la transmisión de valores que contribuyan a formar ciudadanos responsables y comprometidos con el desarrollo sostenible, repensando si es necesario los diseños curriculares por competencias de los grados universitarios. Competencias respecto a las cuales, en este documento, se presenta una tipología centrada en el análisis crítico, la reflexión sistémica, la toma de decisiones colaborativa y la responsabilidad intergeneracional.

En los informes, programas, estrategias y cumbres que han tenido lugar desde hace décadas, hemos querido hacer referencia a varios documentos publicados en los últimos cinco años, bajo el auspicio de Naciones Unidas y la unEsCo. En ellos 
se han planteado importantes retos para iniciar una verdadera transición a la sostenibilidad. Desde sus planteamientos, siempre sujetos a la crítica, se encuentran orientaciones valiosas para participar, desde múltiples instancias, en hacer posible esa transición. En este monográfico que presentamos, hemos querido destacar la responsabilidad y el compromiso adquirido desde la educación para afrontar tales retos desde la acción y la investigación. Como dice Irina Bokova,

No existe una fuerza transformadora más poderosa que la educación para promover los derechos humanos y la dignidad, erradicar la pobreza y lograr la sostenibilidad, construir un futuro mejor para todos, basado en la igualdad de derechos y la justicia social, el respeto de la diversidad cultural, la solidaridad internacional y la responsabilidad compartida, aspiraciones que constituyen aspectos fundamentales de nuestra humanidad común (UNESCO, 2015, 4).

4. LA CUltura DE LA SOSTENiBILIDAD, PUENTE ENTRE LA CULTURA CIENTÍFICA, LA

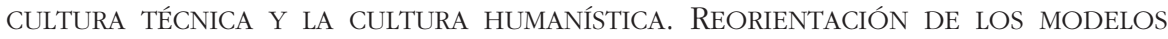
CURRICULARES

La cultura de la sostenibilidad comprende complejas dimensiones, en las que están involucradas estrategias de comportamiento humano y de organización social que son difíciles de formular desde una perspectiva unidimensional. Esta problemática exige soluciones que van más allá de la fragmentación científica y exigen el concurso de áreas de conocimiento tradicionalmente fragmentadas. La evolución del conocimiento científico se ha caracterizado por la fragmentación y el establecimiento de límites entre disciplinas; sin embargo, esta circunstancia es actualmente inviable (Morín, 2001), ya que para abordar los problemas complejos que caracterizan a la sociedad actual no son suficientes ni válidos los instrumentos conceptuales de una disciplina en particular.

El enfoque analítico y plural en el estudio de la realidad incidió en una desmedida preocupación por delimitar y describir el campo científico de cada disciplina, estableciendo fronteras o lindes entre ellas, profundizando analíticamente en el propio campo de estudio y generando una mayor especialización en el conocimiento de su objeto específico. De esta forma las interpretaciones humanísticas, científicas y técnicas de la realidad han ido conformando cadenas paralelas de pensamiento inconexas entre sí.

A finales de la década de los años sesenta, Snow puso de manifiesto de forma explícita (Snow, 1959) la escisión entre la cultura científica y la cultura humanística, que más tarde, a finales del siglo veinte, amplió a la cultura técnica (Snow, 1977). Snow no sólo alertaba de los peligros de dicha escisión, sino que la llegó a considerar como una de las raíces de los desastres de la historia moderna. Esta tesis representa un punto de inflexión respecto a la polarización generalizada entre disciplinas, entre títulos académicos y carreras profesionales; y ha tenido eco en las preocupaciones y ocupaciones de diversos autores por definir "culturas puente" para contrarrestar la fragmentada, compartimentalizada y superespecializada 
manera de ver el mundo (Glotfelty, 2010; Mora Penagos, 2007; Leal Filho, 2009; Arias Rubio, 2015; Morin, 2016).

Esta problemática, objeto de polémica y disensión, ha permitido abrir un campo de reflexión sobre la necesidad de construir una nueva forma de pensar que luche contra la excesiva especialización a la que lleva cada día el conocimiento científico y componga relatos integrados de la realidad que conecten las diferentes cadenas de pensamiento para facilitar una transición hacia la sostenibilidad. Esta cuestión está en el origen, en la década de los años noventa del siglo pasado, de la llamada ciencia de la sostenibilidad, dirigida a desarrollar respuestas efectivas y equitativas a la diversa gama de desafíos que presenta la sostenibilidad, promoviendo una investigación integrada de diferentes áreas académicas (científicas, técnicas y humanísticas) y de diversos equipos académicos, redes, alianzas e instituciones científicas (Komiyama et al., 2006; Vilches et al., 2015).

La educación tiene que encontrar los medios de responder a estos desafíos para reorientar los modelos curriculares, tomando en consideración las diversas cosmovisiones y los sistemas de conocimiento alternativos e integrando el concepto de sostenibilidad en todas las disciplinas. Desde esta óptica, para afrontar el reto de la sostenibilidad son necesarios enfoques inter- y transdisciplinares que propicien transgresiones en las fronteras disciplinarias, que hagan compatibles los tratamientos metodológicos propios de un área científica con las aportaciones metodológicas de otras áreas, desde la lógica de la complejidad, con el objeto de relacionar, contextualizar y globalizar los saberes fragmentados articulando entre sí los diversos ámbitos humanístico, científico y técnico. La sostenibilidad requiere relacionar estos ámbitos como un conjunto integrado para construir conocimiento y aplicarlo a las diferenciadas realidades ambientales, sociales y económicas que de forma integrada inciden en el desarrollo sostenible.

Estas cuestiones subrayan la necesidad de promover la investigación en equipos multidisciplinares que faciliten el desarrollo de modelos curriculares desde un abordaje holístico y posibiliten la transición epistemológica; porque los problemas a los que se enfrentan las sociedades actuales no se pueden solucionar aplicando el mismo tipo de pensamiento que los ha creado.

\section{ESTRUCTURA DEL MONOGRÁFICO}

En este contexto, el monográfico que se presenta trata de poner en valor los trabajos que diferentes equipos de investigación de diversas universidades europeas y americanas y desde diferentes áreas de conocimiento están llevando a cabo para favorecer la transición a la sostenibilidad. Y aborda este tema crucial desde un enfoque interdisciplinario en amplias y variadas facetas. A la luz del resultado de los trabajos presentados, el monográfico se estructura en tres núcleos temáticos:

a) Referentes básicos para una educación orientada a la sostenibilidad.

b) Formación para la sostenibilidad en las instituciones educativas. 
c) Ciudadanía y sostenibilidad.

Todos ellos incluyen un vasto acervo de investigaciones sobre acciones, propuestas y buenas prácticas que se están llevando a cabo en distintas áreas y niveles de la educación. En su conjunto ofrecen un interesante panorama interdisciplinar de los focos que atraen la atención prioritaria de los investigadores que asumen el compromiso educativo con el DHS.

En el primer núcleo temático, se establecen algunos referentes básicos imprescindibles para una educación orientada a la sostenibilidad. Contribuyen al desarrollo de este núcleo los trabajos de M. ${ }^{a}$ Ángeles Murga-Menoyo y María Novo Villaverde (Universidad Nacional de Educación a Distancia) sobre Sostenibilidad, desarrollo "glocal" y ciudadanía planetaria. Referentes de una Pedagogía para el desarrollo sostenible; la aportación de Daniel Gil y Amparo Vilches (Universidad de Valencia) sobre Educación para la Sostenibilidad y Educación en Derechos Humanos: dos campos que deben vincularse, y el artículo de María Casas y Jordi Puig i Baguer (Universidad de Navarra) sobre El impacto ambiental: un despertar ético valioso para la educación.

M. ${ }^{a}$ Ángeles Murga-Menoyo y María Novo se centran en cuestiones teóricas y epistemológicas básicas desde las que afrontar la Educación para el Desarrollo Sostenible. En su trabajo, que ubican en el contexto de un proyecto de investigación financiado por el MINECO, argumentan de modo fundamentado la relación sistémica entre conceptos clave tales como desarrollo glocal y ciudadanía planetaria, que la comunidad científica actual considera relevantes para implementar una educación para el desarrollo sostenible en contextos formales y no formales de enseñanza-aprendizaje. Avanzan en esta dirección desde una visión multiescalar que, según las autoras, significa «asumir como proyecto soluciones planetarias para los problemas globales (destrucción de la naturaleza, migraciones, cambio climático, guerras, hambrunas, escasez de agua potable...), a la vez que implementar respuestas que tendrán su realización en contextos locales». Conciben el desarrollo sostenible en el marco de un modelo glocal y avanzan en la propuesta de un modelo de ciudadanía planetaria enraizada en lo local; desarrollando sus implicaciones para una práctica docente comprometida con la sostenibilidad desde un enfoque sistémico-complejo.

Daniel Gil y Amparo Vilches ponen en evidencia la escasa conexión, tanto en la teoría como en la práctica educativa, entre la sostenibilidad y los derechos humanos, entendidos éstos en su consideración universal, evolutiva y de interdependencia. Defienden que la relación entre una y otros es biyectiva, justificando que el respeto de los derechos humanos es una condición que posibilita la transición a la sostenibilidad, y viceversa, que la transición a la sostenibilidad hace posible el respeto y universalización de los derechos humanos; aunque cabe hacer constar el gran desfase que se produce entre la aprobación y la aplicación de marcos normativos (sociales, culturales y éticos), en función de las dinámicas actuales de poder que inciden en la conculcación de muchos derechos. Los autores presentan documentadas reflexiones sobre la pertinencia de vincular la educación 
en derechos humanos y la Educación para la sostenibilidad como campos de conocimiento y actividad social para reforzar la idea de una ciencia de la sostenibilidad, entendida como el estudio complejo y dinámico de la interacción e interconexión entre los sistemas naturales y humanos con la finalidad de abordar, desde enfoques sistémicos, los problemas glocales que presentan ambos sistemas, tanto desde una perspectiva multidisciplinar, como interdisciplinar y transdisciplinar. Esta cuestión está produciendo avances en el establecimiento de potenciales sinergias entre la ciencia de la sostenibilidad y una educación para el desarrollo sostenible (Mochizuky y Yarime, 2016).

María Casas y Jordi Puig toman como punto de partida para la construcción del artículo la perspectiva del impacto ambiental, como una herramienta con un gran potencial educativo. Presentan un cuidado resumen sobre las principales convenciones, conferencias y declaraciones internacionales que han venido marcando la creciente preocupación por hacer posible una educación para la sostenibilidad. Desde una postura crítica, denuncian la desconexión y desconsideración de la cultura hacia la naturaleza, como parte integrante del núcleo de lo humano y sus graves consecuencias en la fragmentación del saber académico. Defienden una ética de la precaución, acorde con el principio del mismo nombre (Riechmann y Tickner, 2002) para contrarrestar el impacto ambiental, y caracterizan el papel que este impacto puede tener en la educación para el desarrollo sostenible.

El segundo núcleo temático que articula los contenidos de este monográfico se centra en la formación para la sostenibilidad en las instituciones educativas en general y la universidad en particular. En estas diferentes etapas de formación se ha iniciado, a diferentes niveles, un proceso de reflexión estratégica sobre la integración de la Agenda 2030 de Desarrollo Sostenible en los ámbitos de su competencia; lo cual implica abordar sus objetivos de forma transversal en las políticas de formación, abriendo los espacios y generando las condiciones que permitan que la comunidad educativa se implique desde todos sus ámbitos: docencia, investigación y extensión comunitaria. La universidad no constituye sólo un centro de generación de conocimiento, sino que, a través de la formación de los estudiantes, la investigación, la promoción de una conciencia crítica, etc., asume un rol de responsabilidad sin precedentes en la historia de los estudios superiores, de difusión de conocimientos, valores, actitudes y comportamientos en pro de la sostenibilidad, a aplicar por los nuevos titulados universitarios en el ejercicio de sus respectivas profesiones.

Destacan en este núcleo las contribuciones de Lisa Mindt y Marco Rieckmann (University of Vechta, Germany), quienes abordan el Desarrollo de las competencias para el emprendimiento orientado a la sostenibilidad en la educación superior: una revisión bibliográfica de los métodos de enseñanza y aprendizaje; de M. a José Bautista-Cerro y M. ${ }^{a}$ José Díaz (Universidad Nacional de Educación a Distancia), que revisan La sostenibilidad en los grados universitarios: presencia $y$ coherencia, y de Genina Calafell y Mercè Junyent (Universitat Autónoma de 
Barcelona), que se centran en La Idea Vector y sus esferas: una propuesta formativa para la Ambientalización Curricular desde la complejidad.

Lisa Mindt y Marco Rieckman enmarcan su trabajo en el contexto del proyecto europeo CASE, cuya finalidad se dirige al desarrollo de competencias para un emprendimiento que propicie un desarrollo socioeconómico sostenible. La teoría del emprendimiento sostenible busca maximizar convergentemente el beneficio económico, el beneficio social y el beneficio ambiental y defiende el desarrollo de una economía que ponga a las personas y a la naturaleza en el centro del pensamiento económico. Desde esta óptica se trata de introducir en los sistemas educativos competencias para el emprendimiento que propicien el camino hacia la sostenibilidad. Es necesaria una visión integral del proceso de emprendimiento para que se fomente el nacimiento de empresas, además de rentables, respetuosas con el medioambiente y responsables frente a los problemas sociales (Cohen y Winn, 2007). La tesis que se mantiene en la teoría del emprendimiento sostenible estudia el rol que los emprendedores pueden jugar en la creación de una economía productiva, social y ambientalmente sostenible, que pueda superar las barreras para un eficiente funcionamiento de los mercados, contribuir a un eficiente uso de los recursos naturales y al desarrollo de una economía que ponga a las personas y a la naturaleza en el centro del pensamiento económico (Dean y McMullen, 2007). Los autores sitúan su trabajo en el desarrollo de competencias clave necesarias para un emprendimiento sostenible en el ámbito de la educación superior; competencias que no han sido foco de la educación tradicional y que requieren de un alto grado de reflexión individual. Desde esta óptica realizan una revisión bibliográfica sistemática, a partir del análisis de una muestra de 183 artículos publicados en lengua inglesa en los últimos 20 años, y referenciados en dos importantes bases de datos. Centran su análisis sobre los enfoques y métodos de enseñanza y aprendizaje utilizados en el discurso de la educación superior para el desarrollo sostenible y en el discurso de la educación superior para el emprendimiento. Subrayan sus diferencias y la relación de los resultados de aprendizaje con las competencias y la forma en que son evaluadas en ambos ámbitos, con el objetivo de precisar aquellos enfoques y métodos más pertinentes para el emprendimiento orientado a la sostenibilidad. Entre tales propuestas destacan el aprendizaje en escenarios reales, mediante metodologías de aprendizaje activo, reflexivo, colaborativo, transformativo, creativo, aprendizaje-servicio, basado en la resolución de problemas y en proyectos, desde enfoques interdisciplinares.

M. ${ }^{a}$ José Bautista-Cerro y M. ${ }^{a}$ José Díaz realizan una interesante reflexión en torno a la inclusión de la sostenibilidad en 26 titulaciones de los estudios de Grado, tomando como foco de investigación los estudios cursados en la UNED. Se trata de un tema que está siendo objeto de investigación en diversos contextos universitarios europeos con el objetivo de incorporar competencias básicas para la sostenibilidad en la formación universitaria y reorientar los procesos de enseñanza y aprendizaje desde criterios coherentes con los valores de la sostenibilidad. La finalidad es la formación de profesionales comprometidos con el DHs, lo que va 
a requerir la generación de esquemas formativos que integren el medio ambiente natural y social en la actividad profesional de los titulados universitarios. Formar profesionales comprometidos con la sostenibilidad implica cambiar los modelos interpretativos en relación con la protección ambiental, la cohesión y la justicia social y su interacción con la economía, y avanzar hacia modelos alternativos de análisis desde el desarrollo del diálogo disciplinar. El diálogo disciplinar constituye un proceso que debe concretarse en el estudio y análisis de propuestas para afrontar la educación para la sostenibilidad desde una visión que, lejos de ser fragmentada y reduccionista, incorpore los saberes de cada disciplina, estableciendo relaciones articulantes dentro de cada una de ellas. En este trabajo las autoras realizan un análisis cuantitativo y cualitativo de contenido de las memorias de Grado para conocer el nivel de coherencia entre la definición del título, las competencias para la sostenibilidad y su concreción en los resultados de aprendizaje; así como conocer el nivel de presencia de la sostenibilidad en los procesos de formación, como diagnóstico desde el que poder emprender una reorientación pertinente de dichos programas formativos.

Genina Calafell y Mercè Junyent, como miembros del Grupo de investigación Complex, establecen como objetivo de su trabajo impulsar y promover la incorporación de la educación para la sostenibilidad en los centros educativos de enseñanza primaria y secundaria; y proponen, aplican y evalúan un programa formativo basado en el concepto de "Idea Vector» transversal a las áreas de conocimiento, que orienta y vertebra toda la acción educativa, desde una mirada a la complejidad. Según las autoras la «mirada desde la complejidad aporta entender el mundo desde el principio dialógico (entidades aparentemente contrarias pueden dialogar y ser complementarias), el principio sistémico (el todo es más que la suma de las partes) y el principio hologramático (un sistema sigue unas reglas escalares entre lo macro y lo micro en forma de holograma o fractal)». Su propuesta formativa, basada en los principios del modelo ACES (Junyent et al., 2003) define cuatro esferas: la conceptual, que conecta la Idea Vector con los contenidos curriculares de la acción formativa; la creativa, que conecta la Idea Vector con una visión reflexiva de la realidad desde una mirada dialogante entre distintas perspectivas, a veces aparentemente inconexas; la metodológica, que conecta el qué con el cómo para aplicar las estrategias didácticas pertinentes respecto a la Idea Vector; y la esfera de investigación, que conecta la acción educativa con una práctica reflexiva que conduce a investigar en la propia acción. Los resultados obtenidos permiten identificar los factores significativos de un programa formativo dirigido a introducir la sostenibilidad en los currículos y proponer orientaciones para implementar el desarrollo de programas formativos desde la óptica de la sostenibilidad. En este sentido se presentan avances en la implementación de métodos innovadores de enseñanza a partir de la reflexión del profesorado sobre su propia práctica docente.

El tercer núcleo temático gira en torno a la relación entre sostenibilidad y participación ciudadana en el desarrollo comunitario; participación que tiene un componente fundamental de profundización de la democracia. El medio ambiente 
no se puede considerar ya un simple stock de recursos naturales o de materias primas; tampoco las personas son ya simples recursos humanos o mano de obra. Los contextos ambientales y sociales conforman un activo ecosocial que abre nuevas oportunidades y retos que llevan a la necesidad de introducir nuevos valores intangibles y una mayor responsabilidad social en los procesos económicos. La sostenibilidad requiere enfoques de cooperación frente a enfoques de competición, y transcender la idea de un emprendimiento dirigido a la producción económica para generar una idea de emprendimiento para el bien común ${ }^{11}$, anteponiendo a una globalización financiera y empresarial una globalización democrática. Desde esta óptica, el modelo de crecimiento económico tendría que balancearse con el respeto a la calidad ambiental y la preocupación por la paz, la inclusión y la justicia social.

En este núcleo se encuentran las aportaciones de Lucie Sauvé y Hugue Asselin (Université du Québec à Montréal), que abordan el tema de Educar para la Ecociudadanía: contra la instrumentalización de la escuela como antesala del "mercado del trabajo"; José Antonio Caride Gómez (Universidad de Santiago de Compostela), quien centra su trabajo en la Educación social, derechos bumanos y sostenibilidad en el desarrollo comunitario, y Edgar Javier Gonzalez-Gaudiano y Ana Lucía Maldonado-González (Universidad Mexicana de Veracruz), que reflexionan sobre Amenazas y riesgos climáticos en poblaciones vulnerables. El papel de la educación en la resiliencia comunitaria.

Lucie Souvé y Hugue Asselin analizan un proyecto de política nacional, desde un marco de reformas educativas globales, que posee la finalidad de orientar las acciones educativas, centradas fundamentalmente en los aprendizajes instrumentales, hacia el mercado de trabajo, bajo una mirada focalizada esencialmente en el desarrollo económico de contextos específicos, desde un concepto instrumental, competitivo e individualizado del éxito educativo. Las autoras realizan un profundo análisis crítico sobre el significado del desarrollo en las principales iniciativas internacionales (OCDE/PISA, UNECE, 2013; UNESCO, 2014a, 2015, 2016) para examinar sus líneas directrices y estudiar la forma de complementar y enriquecer sus propuestas a la luz de otros ámbitos de reflexión político-educativa. Desde esta óptica proponen una Educación para la ecociudadanía contra la instrumentalización de la educación desde fines político-económicos, cuyo objetivo está puesto en el crecimiento económico para conseguir niveles de vida más avanzados, sin clarificaciones previas de los valores básicos. Partiendo del reconocimiento de las aportaciones de la UNESCO para favorecer un desarrollo humano inclusivo, ambiental y socialmente sostenible, inscriben la ecociudadanía en el campo de la reflexión ética donde los valores no son definidos a priori, sino construidos colectivamente en el crisol de

11. Conclusiones de los «Diálogos universidad y desarrollo sostenible» (CRUE, CUD 2016); documento elaborado por Jorge Solana (UAH) en colaboración con Mar de los Llanos Gómez (UPV) y Nuria Castejón (OCUD). Disponible on line: http://www.ocud.es/es/files/doc870/conclusiones-universidadyods-22.pdf. 
las diferentes situaciones contextuales de interacción. Defienden una educación dirigida al desarrollo integrado de competencias críticas para poner en cuestión las realidades y los discursos establecidos; de competencias éticas que preparen para la búsqueda de los porqués y los para qué como camino para clarificar los propios valores; y de competencias heurísticas que abran las puertas a otros modos de imaginar, creer y pensar. Estos tres campos de competencias preparan de manera convergente para el desarrollo de una competencia política, que es esencial en los procesos de emancipación social y está ligada a la reivindicación y al ejercicio de una democracia ecológica, inclusiva, activa y participativa.

José Antonio Caride, en sintonía con las propuestas contenidas en los últimos documentos de la UNESCO, desarrolla en su trabajo una reflexión sociopedagógica sobre las relaciones y vínculos que se establecen entre la educación social, los derechos humanos y la sostenibilidad desde el prisma del desarrollo comunitario. Los procesos de desarrollo comunitario requieren un sentido bidireccional como condición para su sostenibilidad, por cuanto las personas no han de ser meros objetos de atención, sino sujetos de las decisiones y acciones a desarrollar; lo cual precisa que las propias comunidades locales se impliquen y participen en la toma de decisiones que les afectan tanto en el análisis de los problemas como en la forma de resolverlos. Así, la ciudadanía se convierte en protagonista de su propio desarrollo. Este planteamiento que guarda sinergias con el enfoque del desarrollo endógeno apela según el autor "al empoderamiento de las comunidades y a la descentralización de los mecanismos de poder, ayudando a contextualizar las prácticas educativas y sus aportes al desarrollo local»; prácticas que requieren la adquisición de competencias y capacidades necesarias para que las personas puedan ser agentes activos en la sostenibilidad del desarrollo colectivo. En este trabajo se defiende la promoción de una pedagogía comunitaria en la que el desarrollo y la sostenibilidad sean dos de sus principales referentes, desde los que promover acciones educativas que, siendo congruentes con los principios de calidad ambiental, equidad y justicia social, posibiliten construir una sociedad local-global más democrática, inclusiva y cohesionada.

Finalmente, Edgar González y Ana Lucía Maldonado realizan un estudio dirigido a valorar la percepción del riesgo y de vulnerabilidad de comunidades que, de manera recurrente, son afectadas por fenómenos hidrometeorológicos extremos. Parten de los resultados de estudios previos sobre una encuesta dirigida a estudiantes de centros de enseñanza secundaria de tres localidades veracruzanas afectadas por el riesgo de inundaciones graves que han sufrido en repetidas ocasiones, y centran su trabajo en el análisis de las entrevistas en profundidad realizadas con el personal directivo de dichos centros y con actores clave de las localidades implicadas. Los objetivos van dirigidos a conocer, desde la codificación de cinco categorías de análisis, los problemas y riesgos que afectan a cada localidad, la comunicación preventiva o reactiva ante una inundación, la vulnerabilidad identificada y la resiliencia comunitaria. Los autores establecen como supuesto de partida que, «en los factores sociocognitivos que determinan las disposiciones a actuar por parte 
de poblaciones bajo presiones similares se pueden encontrar claves que permitan interpretar sus reacciones frente a situaciones difíciles». En este artículo se fundamenta un marco teórico muy sólido sobre el concepto de vulnerabilidad y sobre la disposición individual y social de las personas para poner en marcha estrategias, dispositivos y prácticas dirigidas a disminuir y paliar los efectos de la adversidad en sus vidas. Los resultados del trabajo permiten diseñar programas educativos orientados a capacitar a las personas, mediante la definición de criterios y pautas de acción que posibiliten reducir la vulnerabilidad y fortalecer la resiliencia social.

La variedad de voces, enfoques y temas que conforman este número monográfico no obvian la existencia de ausencias, zonas y aspectos insuficientemente representados. Somos plenamente conscientes de la existencia de conceptualizaciones y puntos de vista diversos, necesarios para articular un paradigma que acoja, desde un enfoque holístico, los diferentes avances en los que va discurriendo el compromiso educativo; puesto que en la diversidad está también la gran riqueza del conocimiento. Queremos agradecer a todos los compañeros, en sentido genérico, que abrieron la puerta de Teoría de la Educación. Revista Interuniversitaria a la entrada de este número monográfico, así como a los que, con sus investigaciones, aportaciones y revisiones críticas, han llenado de sentido el contenido del mismo. Esperamos que la estructura que se ha elegido sea útil y ayude a configurar una comprensión amplia y sistemática del conjunto de los temas tratados. Nuestra intención es que los trabajos presentados por los autores contribuyan a profundizar en formas posibles de responder, desde la educación, a los retos planteados por la transición a la sostenibilidad.

\section{REFERENCIAS BIBLIOGRÁFICAS}

ARIAs Rubio, G. (2015) La ambientalización curricular en las humanidades. Tesis Doctoral. Universidad Carlos III, Madrid.

Aznar Mínguet, P. y Ull Solís, M. ${ }^{a}$ A. (2012) La responsabilidad por un mundo sostenible. Propuestas educativas a padres y profesores. Bilbao, Desclée de Brouwer.

Banco Mundial (1987) Environment, Growth, and Development. Washington D.C., World Bank, Development Committee, Pamphlet 14.

Barbiroli, G. (ed.) (2009) Principles of sustainable development. Oxford, EOlss Publishers/ UNESCO.

BARRÓn RUIZ, A. (2002) Ética ecológica y educación ambiental en el siglo XXI, en HERnÁndez, J. M. et al. (eds.) La educación y el medio ambiente natural y humano. Salamanca, Ediciones Universidad de Salamanca, 21-37.

Barth, M.; Michelsen, G.; Rieckmann, M. y Thomas, I. (eds.) (2016) Routledge Handbook of Higher Education for Sustainable Development. New York, Routledge International Handbooks.

Bauman, Z. (2007) Tiempos líquidos. Barcelona, Tusquets.

BECK, U. (2008) La sociedad del riesgo: en busca de la seguridad perdida. Barcelona, Paidós Ibérica.

Bustelo, P. (1998) Teorías contemporáneas del desarrollo económico. Madrid, Síntesis. 
CARIDE, J. A. y MeIrA, P. A. (2001) Educación ambiental y desarrollo bumano. Barcelona, Ariel. Chenery, H. M. S. et al. (1976) Redistribución con Crecimiento. Madrid, Tecnos y Banco Mundial.

Cohen, B. y Winn, M. (2007) Market imperfections, opportunity and sustainable entrepreneurship. Journal of Business Venturing, 22, 29-49.

Colom, A. J. (2007) La complejidad del desarrollo sostenible. Miscelánea Comillas. Revista de Ciencias Humanas y Sociales, 65 (126), 511-534

Cortina, A. y Pereira, G. (2009) Pobreza y libertad. Erradicar la pobreza desde el enfoque de Amartya Sen. Madrid, Tecnos.

Dale, A. (2001) At the Edge. Sustainable Development in the 21st Century. Vancouver, University of British Columbia Press.

Daly, H. E. y COBB, J. (1989) For the common god. Redirecting the economy toward community, the environment, and a sustainable future. Boston, Beacon Press.

Dean, T. y McMullen, J. (2007) Toward a theory of sustainable entrepreneurship: Reducing environmental degradation through entrepreneurial action. Journal of Business Venturing, 22, 50-76.

Delors, J. (1996) La Educación encierra un tesoro. París, unesco.

Diamond, J. (2006) Colapso. Barcelona, Debate.

Doyal, L y Gough, I. (1994) Teoría de las necesidades humanas. Barcelona, Icaria-Fuhem.

DuARTE, C. (coord.) (2006) Cambio Global. Impacto de la actividad humana sobre el sistema Tierra. Madrid, CSIC.

ELIZALDE, A. (2009) ¿Qué desarrollo puede llamarse sostenible en el siglo XXI? La cuestión de los límites y las necesidades humanas. Revista de Educación. Número extraordinario, 53-75.

Ezechieli, E. (2003) Beyond Sustainable Development: Education for Gross National Happiness in Bhutan. Redwood City, Stanford University Press.

FAuRE, E. (1972) Aprender a ser. París, UnESCO.

FELBER, Ch. (2012) La economía del bien común. Barcelona, Deusto.

FERnÁNDEZ FERnÁNDEZ, J. L. (2004) Finanzas y ética. La dimensión moral de la actividad financiera y el Gobierno Corporativo. Madrid, Universidad Pontificia Comillas.

García, E, (2004) Medio ambiente y sociedad: la civilización industrial y los límites del planeta. Madrid, Alianza Editorial.

Glotfelty, C. (2010) Los estudios literarios en la era de la crisis medioambiental, en Flysl, C.; Marrero, J. M. y Barella, J. (eds.) Ecocríticas: Literatura y medio ambiente. Madrid, Iberoamericana-Vervuert.

Gudynas, E. (2004) Ecología, economía y ética del desarrollo sostenible. Montevideo, Ed. Coscoroba.

HABERMAS. J. (2008) La postmodernidad. Barcelona, Kairós.

Helliwell, J.; Layard, R. y SACHS, J. (2017) World Happiness Report 2017. New York, Sustainable Development Solutions Network.

Hirschman, A. O. (1980) Auge y ocaso de la teoría económica del desarrollo. El Trimestre Económico, 188, 1055-1077.

Hopwood, B.; Mellor, M. y O'Brien, G. (2005) Sustainable development: mapping different approaches. Sustainable Development, 13 (1), 38-52.

Jackson, T. (2011) Prosperidad sin crecimiento. Economía para un planeta finito. Barcelona, Icaria Ed.

Jordan, A. y AdGER, N. (eds.) (2009) Goberning Sustainability. Cambridge, Cambridge University Press. 
KomiYama, H. y TAKeuchi, K. (2006) Sustainability Sciences: building a new discipline. Sustainability Sciences Review, 1 (1), 1-6.

LAMBERTON, G. (2005) Sustainable sufficiency-an internally consistent version of sustainability. Sustainable Development, 13 (1), 53-78.

Latouche, S. (2007) Sobrevivir al desarrollo. Barcelona, Icaria.

LEAL FILHO, W. (2009) La educación para la sostenibilidad: Iniciativas internacionales. Revista de Educación (1), 263-277.

LÓPEZ, V. (2008) Sustentabilidad y desarrollo sustentable: origen, precisiones conceptuales y metodología operativa. Mexico, Editorial Trillas.

Lyotard, J. F. (1989) La condición postmoderna. París, Éditions de Minuit.

MAX-NeEF, M. (1993) Desarrollo a escala humana: conceptos, aplicaciones y reflexiones. Barcelona, Icaria Ed.

Meadows, D. H.; Meadows, D. L.; Randers, J. y Behrens, W. (1972) Los límites del crecimiento. México, Fondo de Cultura Económica.

Meadows, D. H.; Randers, J. y Meadows, D. L. (2006) Los límites del crecimiento: 30 años después. Barcelona, Galaxia Gutenberg.

Mora Penagos, W. M. (2007) Respuesta de la universidad a los problemas socio-ambientales: la ambientalización del currículo en la educación superior. Revista Investigación en la Escuela, (63), 65-76.

Morin, E. (2001) Los siete saberes necesarios para la educación del futuro. Barcelona, Paidós.

MORIN, E. (2011) La vía para el futuro de la humanidad. Barcelona, Paidós.

MORIN, E. (2016) Enseñar a vivir. Manifiesto para cambiar la educación. Barcelona, Paidós.

MUCHIZUKI, Y. y YARIME, M. (2016) Education for sustainable development and sustainability Science: Re-purposing higher education and research, en BARTH, M.; Michelsen, G.; Rieckmann, M. y Thomas, I. (eds.) Routledge Handbook of Higher Education for Sustainable Development. New York, Routledge International Handbooks.

Murga-Menoyo, M. Á. (2009) La Carta de la Tierra. Un referente de la Década por la Educación para un Desarrollo Sostenible. Revista de Educación, n. ${ }^{\circ}$ extraordinario, 239-262.

Murga-Menoyo, M. Á. (2015) Desarrollo sostenible. Problemáticas, agentes y estrategias. Madrid, McGraw Hill/unED.

Naredo, J. M. (2006) Raíces económicas del deterioro ecológico y social: más allá de los dogmas. Madrid, Siglo XXI.

Novo, M. (2006) El desarrollo sostenible. Su dimensión ambiental y educativa. Madrid, Pearson/UNESCO.

Nussbaum, M. (2002) Las mujeres y el desarrollo bumano: el enfoque de las capacidades. Barcelona, Herder.

ONu (2015) Transformar nuestro mundo: la Agenda 2030 para el desarrollo sostenible. Nueva York. A/69/L.85.

pnud (Programa de Naciones Unidas para el Desarrollo) (1990) Informe desarrollo humano. Consultado el 5 de abril de 2017. http://hdr.undp.org/es/content/ informe-sobre-desarrollo-humano-1990.

PNUD (1993) Informe desarrollo bumano. Consultado el 5 de abril de 2017. http://hdr.undp. org/es/content/informe-sobre-desarrollo-humano-1993.

PNUD (1994) Informe desarrollo humano. Consultado el 5 de abril de 2017. http://hdr.undp. org/es/content/informe-sobre-desarrollo-humano-1994. 
PNUD (2016) Informe desarrollo humano. Consultado el 5 de abril de 2017. http://www. undp.org/content/undp/es/home/librarypage/hdr/2016-human-development-report. html.

Rieckmann, J. (2005) Un mundo vulnerable. Ensayos sobre ecología, ética y tecnociencia. Madrid, Los Libros de la Catarata.

Riechmann, J. (coord.) (2006) Perdurar en un planeta habitable. Ciencia, tecnología y sostenibilidad. Barcelona, Icaria.

Riechmann, J. y Tickner, T. (2002) El principio de precaución. Barcelona, Icaria.

Rostow, W. (1961) Las etapas del crecimiento económico. Un manifiesto no comunista. México, Fondo de Cultura Económica.

SACHS, J. (2008) Economía para un planeta abarrotado. Barcelona, Debate.

SEN, A. K. (1995) Nueva economía del bienestar. Valencia, Universidad de Valencia, Servicio de Publicaciones

Sen, A. K. (2000) Desarrollo y libertad. Barcelona, Planeta.

Sen, A. K. y KliksBerg, B. (2007) Primero la gente: Una mirada desde la ética del desarrollo a los principales problemas del mundo globalizado. Barcelona, Ediciones Deusto.

SnOw, C. P. (1959) The Two Cultures and the scientific revolution. Cambridge, University Press.

SNOw, C. P. (1977) Las dos culturas y un segundo enfoque. Madrid, Alianza Editorial.

Stiglitz, J. D. (2006) Cómo hacer que funcione la globalización. Madrid, Taurus.

Stiglitz, J. D. y Charlton, A. (2007) Comercio justo para todos. Madrid, Taurus.

SuTcliffe, B. (1995) Desarrollo frente a Ecología. Revista de Ecología Política, 9, 27-49.

Tilbury, D. (2011) Educación para el desarrollo sostenible: examen por los expertos de los procesos y el aprendizaje. París, unesco. Consultado el 20 de abril de 2017. http:// unesdoc.unesco.org/images/0019/001914/191442s.pdf.

UNECE (2011) Strategy for Education for Sustainable development. ECE/CEP/AC13/2011/6.

UNECE (2013) Empowering educators for a sustainable future. Tools for policy and practice workshops on competences in education for sustainable development. ECE/CEP/165 AC.13/2013/4. GE.13-20120.

unESCO (2014a) Shaping the Future We Want. UN Decade of Education for Sustainable Development (2005-14). Final Report. Consultado el 20 de abril de 2017. http://unesdoc. unesco.org/ images/0023/002301/230171e.pdf.

unESCO (2014b) Declaración sobre la Educación para el Desarrollo Sostenible. Aichy-Nagoya (Japón). ED/2014/TLC/ESD/01.

UNESCO (2015) Replantear la educación: ¿hacia un bien común mundial? París, UNESCO.

Vattimo, G. (1986) El fin de la modernidad. Nibilismo y bemenéutica en la cultura postmoderna. Barcelona, Gedisa.

Vilches, A. y GIL, D. (2015) Ciencia de la sostenibilidad: ¿una nueva disciplina o un nuevo enfoque para todas las disciplinas? Revista Iberoamericana de Educación, 69 (1), 39-60.

Vilches, A.; MACíAs, O. y GiL, D. (2014) La transición a la sostenibilidad: un desafío urgente para la ciencia, la educación y la acción ciudadana. Colección de documentos de trabajo de Iberciencia. Temas clave de reflexión y acción. Consultado el 27 de abril de 2017. http://www.ibercienciaoei.org/documentoiberciencia1.pdf.

wCed (World Commision on ENvironment and Development) (1987) Our Common Future (Brundtland Report). United Nations. Consultado el 20 de abril de 2017. https:// es.scribd.com/doc/105305734/ONU-Informe-Brundtland-Ago-1987-Informe-de-la-Comision-Mundial-sobre-Medio-Ambiente-y-Desarrollo. 